\title{
Dynamic correlations in a charged Bose gas
}

\author{
K. Tankeshwar \\ Center for Advanced Study in Physics, Department of Physics, Panjab University, 160014 Chandigarh, India \\ B. Tanatar \\ Department of Physics, Bilkent University, Bilkent, 06533 Ankara, Turkey \\ M. P. Tosi* \\ International Centre for Theoretical Physics, I-34100 Trieste, Italy
}

(Received 31 July 1997)

\begin{abstract}
We evaluate the ground-state properties of a charged Bose gas at $T=0$ within the quantum version of the self-consistent field approximation of Singwi, Tosi, Land, and Sjölander. The dynamical nature of the localfield correction is retained to include dynamic correlation effects. The resulting static structure factor $S(q)$ and the local-field factor $G(q)$ exhibit properties not described by other mean-field theories. [S0163-1829(98)06612-0]
\end{abstract}

\section{INTRODUCTION}

The homogeneous gas of electrons interacting via the Coulomb potential is a useful model ${ }^{1}$ to understand a vast range of physical phenomena. The continuing interest in this model stems partly from the realization of physical systems in the laboratory that lend themselves to such a description, and partly from theoretical reasons to understand the basic properties of a many-body system. Similarly, a system of charged particles obeying Bose statistics under the influence of Coulomb interactions is important in determining the effects of statistics and correlations in comparison with the electron gas. Charged bosons are the subject of renewed interest because of their possible role in some high-temperature superconducting systems ${ }^{2}$ and in astrophysical applications. ${ }^{3}$

In the study of many-body properties of charged particles, the self-consistent field method of Singwi et al. ${ }^{4}$ (STLS) provides a means of going beyond the random-phase approximation (RPA) in a simple and physically motivated way. It has been successfully applied to electron gas systems in various situations including different dimensions ${ }^{4,5}$ and also to the charged Bose gas. ${ }^{6,7}$ The local-field factor introduced in the STLS scheme to describe the correlation effects depends on the wave vector only. This is because classical distribution functions were used in its original derivation. A quantum version of the STLS approach (qSTLS) was developed by Hasegawa and Shimizu ${ }^{8}$ that allows for a frequencydependent local-field factor. A different formulation (with similar results) was put forward by Niklasson, ${ }^{9}$ who also elucidated the relations among various related approximations. Numerical calculations on the self-consistent equations for a three-dimensional (3D) electron gas were provided by Holas and Rahman. ${ }^{10}$ A detailed corresponding study in a 2D electron liquid has recently appeared. ${ }^{11}$ Schweng et al.. ${ }^{12}$ have investigated the frequency dependence of $G(q, \omega)$ within a finite-temperature formalism. The main finding of all these efforts has been that the quantum effects embodied in $G(q, \omega)$ change significantly the short-range correlations.

In this paper, we apply the qSTLS method to the study of a charged Bose gas in three dimensions. Our main motivation, apart from possible applications, is to test for charged bosons the efficiency of the qSTLS method, which is demonstrated to yield accurate results for electron systems. We compare our results with more elaborate hypernetted chain (HNC) calculations ${ }^{13}$ and with quantum Monte Carlo (QMC) results. ${ }^{14,15}$ Both in the HNC calculations and in the QMC simulations static local-field corrections have been extracted. In this work our aim is to investigate the dynamical nature of $G(q, \omega)$. The frequency dependence of the dynamic $G(q, \omega)$ has recently been emphasized in some theories dealing with superconductivity. ${ }^{16}$

The paper is organized as follows. In the next section we outline the qSTLS method. In Sec. III we present our selfconsistent calculations, compare the results with other works, and discuss the effects of $G(q, \omega)$ on certain physical quantities. We conclude with a brief summary in Sec. IV. In the Appendix, we provide some technical details on the calculation of $G(q, \omega)$.

\section{THEORY}

We consider a system of negatively charged bosons embedded in a uniform positive background, interacting via the Coulomb potential. The system is characterized by the dimensionless coupling constant $r_{s}=r_{0} / a_{B}$, where $r_{0}=(3 / 4 \pi n)^{1 / 3}$ is the average interparticle spacing, $a_{B}=1 / m e^{2}$ is the Bohr radius, and $n$ is the number density (we use $\hbar=1$ ). The bare Coulomb interaction is given by $V(q)=4 \pi e^{2} / q^{2}$, and at the lowest order (in the Bogoliubov approximation) the static structure factor of the system is $S(q)=\left[1+2 n V(q) / \epsilon_{q}\right]^{-1 / 2}$, where $\epsilon_{q}=q^{2} / 2 m$ is the freeparticle energy. Using the Feynman expression for the excitation spectrum we can determine the collective modes of the system to be $\omega_{\mathrm{pl}}=\left[\epsilon_{q}^{2}+2 n \epsilon_{q} V(q)\right]^{1 / 2}$. The lowest-order theory (also known as the uniform limit) neglects correlation effects, which become increasingly important at large coupling strength.

In the STLS approximation the density-density response 
function is given in the form of a generalized random-phase approximation (RPA),

$$
\chi(q, \omega)=\frac{\chi_{0}(q, \omega)}{1-V_{\mathrm{eff}}(q, \omega) \chi_{0}(q, \omega)},
$$

where $\chi_{0}(q, \omega)$ is the zero-temperature dynamic susceptibility of a noninteracting Bose gas

$$
\chi_{0}(q, \omega)=\frac{2 n \epsilon_{q}}{(\omega+i \eta)^{2}-\epsilon_{q}^{2}},
$$

defined in terms of the density $n$ and free-particle energy $\epsilon_{q}$ ( $\eta$ is a positive infinitesimal quantity). The effective interaction $V_{\text {eff }}(q, \omega)=V(q)[1-G(q, \omega)]$ is defined in terms of the dynamic (frequency-dependent) local-field factor

$$
G(q, \omega)=-\frac{1}{n} \int \frac{d \mathbf{q}^{\prime}}{(2 \pi)^{3}} \frac{\chi_{0}\left(\mathbf{q}, \mathbf{q}^{\prime} ; \omega\right)}{\chi_{0}(q, \omega)} \frac{V\left(q^{\prime}\right)}{V(q)}\left[S\left(q-q^{\prime}\right)-1\right],
$$

for which we give an explicit formula in the Appendix. In the above expression for $G(q, \omega)$, the static structure factor $S(q)$ is related to the dynamical susceptibility through the fluctuation-dissipation theorem

$$
S(q)=-\frac{1}{n \pi} \int_{0}^{\infty} d \omega \chi(q, i \omega),
$$

where we have used the analytic continuation of the response function to the complex frequency plane followed by the Wick rotation of the frequency integral. This procedure is more effective in capturing the plasmon poles dominating the response of a Bose fluid.

The derivation leading to the expression for the frequency-dependent local-field factor $G(q, \omega)$ is similar to that in the static STLS approximation. Instead of using the classical distribution functions, a set of equations of motion for the Wigner distribution functions are considered. The hierarchy of coupled equations are terminated by making the assumption that the two-body Wigner distribution function may be written as a product of one-body distribution functions and the pair-correlation function $g(r) .{ }^{8,10}$ The frequency dependence of $G(q, \omega)$ comes from the factors $\chi_{0}\left(\mathbf{q}, \mathbf{q}^{\prime} ; \omega\right)$ and $\chi_{0}(q, \omega)$, which are the inhomogeneous and homogeneous free-particle response functions, respectively. We give the definition of $\chi_{0}\left(\mathbf{q}, \mathbf{q}^{\prime} ; \omega\right)$ and a simple quadrature formula for $G(q, i \omega)$ (evaluated on the imaginary frequency axis) in the Appendix. We iterate between $G(q, i \omega)$ and $S(q)$, which uses $\chi(q, i \omega)$ and in turn $G(q, i \omega)$, until self-consistency is achieved.

It is known that the STLS approach fails to fulfill the compressibility sum rule, namely the compressibility evaluated directly from the ground-state energy is not equal to that calculated using the long-wavelength limit of $G(q, 0)$. This deficiency may be overcome within a construction given by Vashishta and Singwi ${ }^{17}$ and may be applied to the present problem of dynamic local-field corrections as shown by Holas and Rahman. ${ }^{10}$ Since our primary aim is to investigate the qualitative effects of the frequency dependence of $G(q, \omega)$, we consider only the qSTLS scheme.
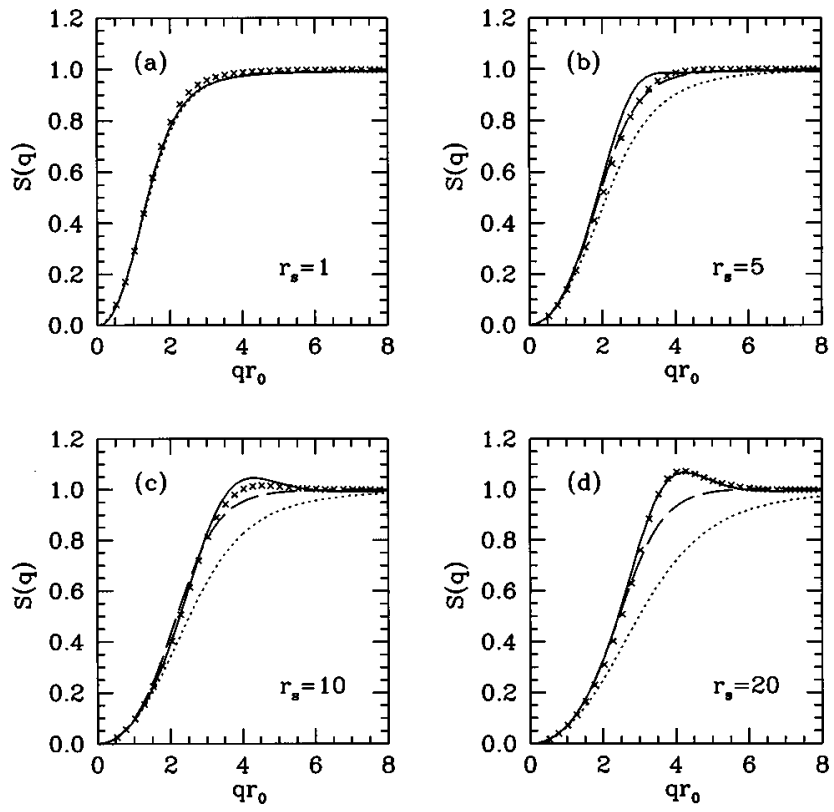

FIG. 1. (a) The static structure factor $S(q)$ in a charged Bose gas at $r_{s}=1$. Solid and dotted lines show the qSTLS and RPA results, respectively. Crosses are the QMC simulation results from Ref. 15. (b) - (d) $S(q)$ at $r_{s}=5,10$, and 20, respectively. In (b)-(d) the dashed lines are the static STLS results from Ref. 6.

\section{RESULTS AND DISCUSSION}

Since the dynamic local field factor depends on the static structure factor within the qSTLS approximation, and the latter depends on the former through the fluctuationdissipation integral, they have to be calculated selfconsistently. Although the frequency dependence of $G(q, \omega)$ makes the calculation slightly more demanding than the usual STLS method, ${ }^{6,7}$ it is still manageable and we performed calculations for several densities until convergence was achieved to an accuracy of $0.01 \%$.

We first discuss the static structure factor resulting from our self-consistent calculations. The static structure factor of the 3D charged Bose system is shown in Fig. 1, for various $r_{s}$ values. The correlation effects (treated here dynamically) induce a vast difference with increasing $r_{s}$ compared to the RPA results [when $G(q, \omega)=0$ ] and the static STLS results. ${ }^{6}$ At high densities (small $r_{s}$ ) $S(q)$ is similar to that obtained within the static STLS approximation. ${ }^{6,7}$ As $r_{s}$ increases a peak structure starts to appear with increasing amplitude. Such peaks in the static structure factor were observed in the calculations of Apaja et al. ${ }^{13}$ in which the hypernetted-chain approximation was used, and in the Monte Carlo simulations of Moroni et al. ${ }^{15}$ We attribute the peak structure in $S(q)$ to the inclusion of dynamic correlation effects. The static STLS calculations of Conti et al. ${ }^{7}$ also show the emergence of a peak in $S(q)$ at large $r_{s}$, when the self-consistency condition on the compressibility sum rule is imposed.

In Fig. 2 the probability of finding a particle at distance $r$ away from a particle situated at the origin, namely the pair distribution function $g(r)$ is shown for several $r_{s}$ values. We use the Fourier transform

$$
g(r)=1+\frac{1}{2 \pi^{2} n r} \int_{0}^{\infty} d k k \sin (k r)[S(k)-1]
$$



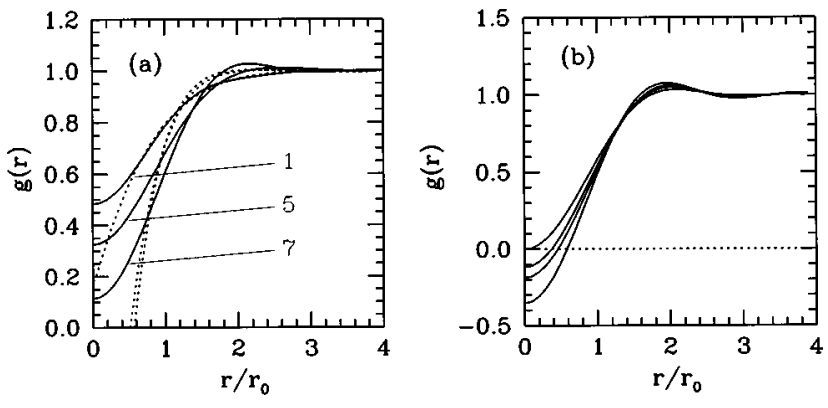

FIG. 2. (a) The pair-correlation function $g(r)$ as a function of $r$. Curves from top to bottom are for $r_{s}=1,5$, and 7, respectively. Dotted lines are the RPA results. (b) $g(r)$ at lower density. Curves from top to bottom as they appear on the ordinate indicate $r_{s}=10$, 20,30 , and 50, respectively.

to find that $g(r)$ remains positive for $r_{s} \lesssim 12$. This is a large improvement over the RPA (or Bogoliubov) result. The $g(r)$ within the present qSTLS approach starts to become negative for small values of $r$ as the coupling strength increases. We point out, however, that in the present scheme only the dynamics of the Pauli correlation hole is taken into account, but not the Coulomb correlation hole, which becomes dominant at low densities. ${ }^{11}$

From our self-consistent calculations of the correlation effects we obtain the frequency-dependent local-field factor. Figure 3 shows $G(q, i \omega)$ as a function of frequency on the imaginary axis. We note that $G(q, i \omega)$ is a smooth function of $\omega$, tending to a constant for fixed values of $q$, and most of the frequency dependence is confined to the low-frequency region. The QMC simulations can only reveal information on the static local field, thus our frequency dependent results for $G(q, \omega)$ present a different aspect of the correlation effects.

The real and imaginary parts of $G(q, \omega)$ may be obtained from $G(q, i \omega)$ by the analytic continuation $i \omega \rightarrow \omega+i \eta$ in Eq. (3). We show in Fig. 4 the real and imaginary parts of $G(q, \omega)$ as functions of frequency at $r_{s}=10$ and $q r_{0}=2,4$, and 6. We observe that both the real and imaginary parts of $G(q, \omega)$ oscillate as a function of $\omega$. One can show that for fixed $q$ and large $\omega$, the local-field factor behaves as

$$
\lim _{\omega \rightarrow \infty} G(q, \omega)=G_{\mathrm{STLS}}(q)+\mathcal{O}\left(1 / \omega^{2}\right),
$$

a property also known to exist in electron fluids. ${ }^{10}$ Thus, $\operatorname{Im}[G(q, \omega)]$ vanishes and $\operatorname{Re}[G(q, \omega)]$ tends to the value
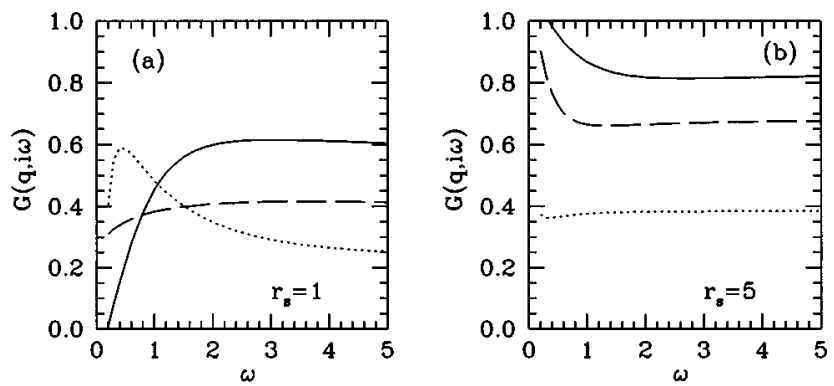

FIG. 3. (a) The local-field factor evaluated on the imaginary frequency axis as a function of $\omega$ (in units of $1 / 2 m r_{0}^{2}$ ) at (a) $r_{s}=1$ and (b) $r_{s}=5$. Dotted, dashed, and solid lines are for $q r_{0}=2,4$, and 6 , respectively.
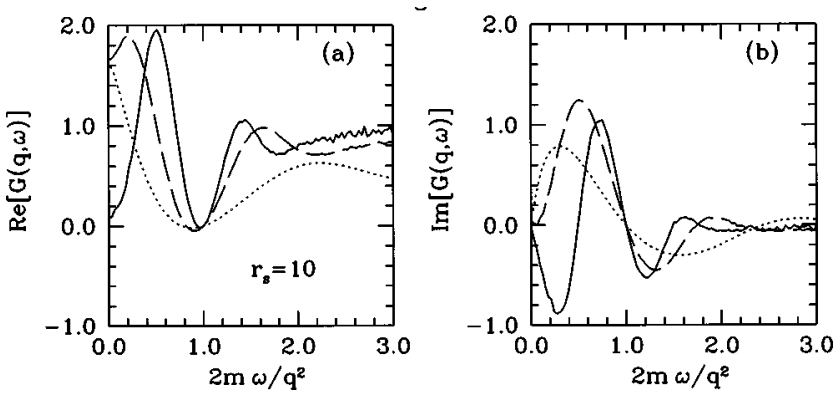

FIG. 4. (a) The real part of $G(q, \omega)$ as a function of frequency at $r_{s}=10$ and $q r_{0}=2$ (dotted line), $q r_{0}=4$ (dashed line), and $q r_{0}=6$ (solid line). (b) Same as (a) for the imaginary part of $G(q, \omega)$.

given by the static STLS approximation for large $\omega$, as illustrated in Fig. 4. In the static limit, i.e., $\omega=0, \operatorname{Im}[G(q, 0)]$ vanishes, and the real part satisfies $\lim _{q \rightarrow \infty} G(q, 0)$ $=1-g(0)$.

The zero-frequency limit $G(q, 0)$ is of interest in most practical applications. Within the dynamical STLS theory $G(q, 0)$ is given by

$$
G(q, 0)=\frac{q^{3}}{3 \pi} \int_{0}^{\infty} d k \frac{k}{\left(q^{2}-k^{2}\right)} \ln \left|\frac{q-k}{q+k}\right|[S(k)-1] .
$$

In Fig. 5 we employ the above expression using our selfconsistent $S(q)$ (solid lines) and that coming from the QMC simulations ${ }^{15}$ (dashed lines). We observe that both calculations agree quantitatively for $q r_{0} \lesssim 2$ and qualitatively for $q r_{0} \gtrsim 2$. The differences originate from the respective structure factors. $G(q, 0)$ calculated in this manner is very different from the results of static STLS approximation ${ }^{6}$ and also from $G(q)$ of QMC simulations. ${ }^{15}$ We note that in the QMC simulations $G(q)$ is evaluated directly by the response of the system to an external perturbation, without using the static structure factor $S(q)$. It remains interesting that $G(q, 0)$ for
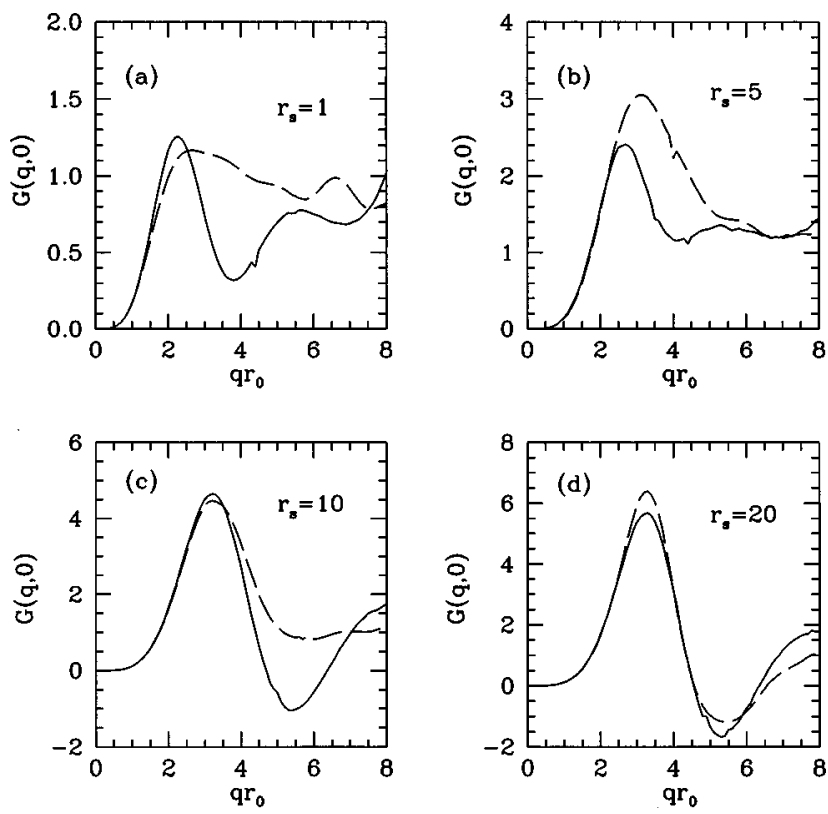

FIG. 5. (a) -(d) The local-field factor $G(q, 0)$ at $r_{s}=1,5,10$, and 20 , calculated within the dynamical theory with self-consistent $S(q)$ (solid lines) and QMC-S( $q$ ) (dashed lines). 


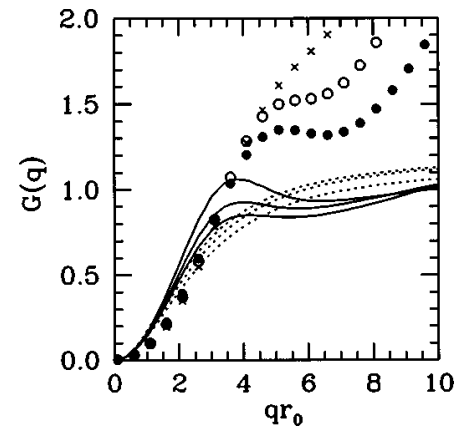

FIG. 6. $G(q)$ in various approximations. Crosses, open circles, and solid circles indicate the QMC $G(q)$ at $r_{s}=10,20$, and 50, respectively. Solid lines are the present results of $G(q, \omega=\infty)$ from bottom to top at the same $r_{s}$ values. Dotted lines are the static STLS results of Ref. 6 .

charged bosons displays rather different behavior than the QMC results, whereas in the electron gas case ${ }^{10}$ it provides a meaningful estimate.

The high-frequency limit of our $G(q, \omega)$ also yields a frequency-independent local-field factor $G_{\infty}(q)$. Our selfconsistent results for $G_{\infty}(q)$ are compared with the QMC and static STLS approximations in Fig. 6. We observe in Fig. 6 that the qSTLS approximates the local field factor at small $q$, but is not able to reproduce the large- $q$ behavior of the QMC results. This is not surprising since the large- $q$ nature of $G(q)$ mainly comes from the momentum distribution (or kinetic energy of the interacting system), which we have assumed to be a $\delta$ function. However, our results capture part of the essential features seen in the more precise theories. ${ }^{13,15}$ For instance, the peak structure in $G_{\infty}(q)$ can be identified as in the QMC and $\mathrm{HNC}$ calculations around $q r_{0} \approx 4$, where the first star of the reciprocal lattice of the body-centered cubic crystal would lie. STLS-type mean-field theories without the frequency dependence ${ }^{6}$ tend to display a monotonic behavior in $G(q)$. Thus, it appears that our $G_{\infty}(q)$ may be useful as a good estimate of the true $G(q, 0)$, i.e., as extracted from QMC simulations.

In Fig. 7 we plot the plasmon dispersion curve $\omega_{q}$ scaled with the long-wavelength plasmon energy $\omega_{\mathrm{pl}}=\left(12 r_{s}\right)^{1 / 2}$ at various $r_{s}$ values. $\omega_{q}$ is obtained by solving for the pole of the dynamic susceptibility given in Eq. (1), which includes the frequency-dependent local-field factor. We compare our results with the plasmon dispersion in the RPA $\left[\omega_{q}^{\mathrm{RPA}}=\left(12 r_{s}\right)^{1 / 2}\left(1+q^{4} / 12 r_{s}\right)^{1 / 2}\right]$ and with the static STLS calculations. ${ }^{6}$ For small $q$, the plasmon dispersion obtained from the present dynamical theory is very similar to that of static STLS. The roton minimum appearing at intermediate $q$, on the other hand, is more pronounced and shifted towards the low- $q$ side. At large $q$ the qSTLS results approach the RPA faster than the static STLS. The differences between the qSTLS and the static STLS approximations become more marked with increasing $r_{s}$. Since our frequency-dependent local-field factor has an imaginary part, we also calculate the damping $\gamma_{q}$, of the plasma excitations by solving for the imaginary part of the equation,

$$
1-V(q)\left[1-G\left(q, \omega_{q}-i \gamma_{q}\right)\right] \chi_{0}\left(q, \omega_{q}-i \gamma_{q}\right)=0 .
$$

Carrying out a standard analysis, ${ }^{18}$ which assumes that $\gamma_{q}$ $\ll \omega_{q}$, we find that the damping is given by

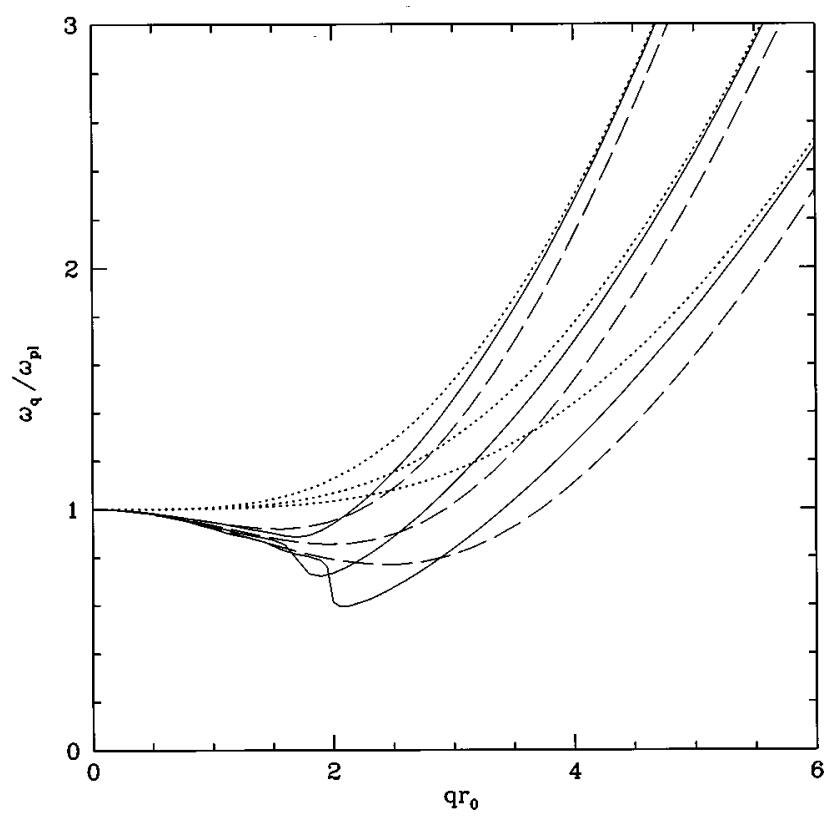

FIG. 7. The plasmon dispersion relation $\omega_{q}$ scaled with respect to the long-wavelength plasmon energy as a function of $q$. Solid curves from top to bottom are for $r_{s}=5,10$, and 20, respectively. Dashed and dotted lines are the corresponding results of the static STLS approximation from Ref. 6 and of the RPA, respectively.

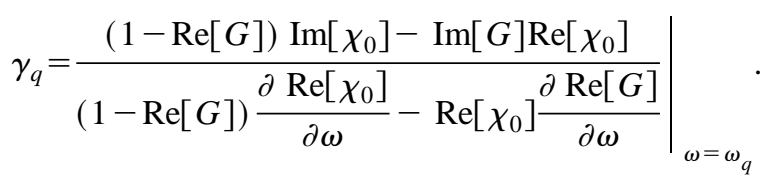

$\gamma_{q}$ calculated according to the above expression is of the order of $0.5 \times 10^{-3} \omega_{\mathrm{pl}}$ for $q r_{0} \lesssim 2.5$.

In general, the excitation spectrum of a charged Bose gas contains multipair excitations ${ }^{1}$ in addition to the plasmon mode. Our approach neglects multiparticle correlation effects since the lowest-order polarization diagram described by $\chi_{0}(q, \omega)$ is included in the calculation of $G(q, \omega)$. The role of multiparticle effects in the excitation spectrum as described by the dynamic structure factor $S(q, \omega)$ has been thoroughly discussed by Apaja et al. ${ }^{13}$

It has been argued ${ }^{19,10}$ that when the static local-field factor $G(q, 0)$ becomes greater than unity, the system of charged particles may exhibit the formation of a chargedensity wave (CDW). The instability will set in at a critical $r_{s}$ value when

$$
G(q, 0)=1+\frac{1}{V(q)\left|\chi_{0}(q, 0)\right|},
$$

which may be derived from the condition $1 / \varepsilon(q, 0) \leqslant 1$, for the static dielectric function. Since the accurate QMC results $^{14,15}$ show no evidence of the violation of the above inequality, it may be concluded that the charged bosons remain in a stable fluid phase until crystallization occurs.

We have uncritically assumed that the charged Bose gas is in the condensate state and determined the effects of correlations induced by Coulomb interaction, as in previous calculations of a similar nature. ${ }^{6,7}$ However, the QMC simulations ${ }^{15}$ indicate that even at relatively high density 
(weak coupling) only about $80 \%$ of the particles are in the condensate. The interactions enormously deplete the condensate so that by $r_{s}=160$ (crystallization density), most of the particles occupy nonzero momentum states. Thus, the results of approximate theories such as STLS or qSTLS should be used with caution at large $r_{s}$ values. It might be possible to account for the effects of the particles out of the condensate by choosing a suitable model for the distribution function (say, a Gaussian) that determines the response of the noninteracting system. These ideas need to be explored within the self-consistent scheme for quantitative assessment.

\section{SUMMARY}

We have considered the system of charged bosons at $T=0$ and studied the effects of interactions within the quantum version of the STLS scheme. The self-consistently calculated static structure factor $S(q)$ exhibits a peak around $q r_{0} \approx 4$ with increasing $r_{s}$, in agreement with QMC and HNC calculations. The local-field factor $G(q, \omega)$ is frequency dependent in the present approximation and reveals information about dynamical correlations. In particular, inclusion of the dynamic $G(q, \omega)$ improves the static STLS results in predicting the $r_{s}$-dependent behavior of $G(q)$ for wave vectors $q r_{0} \lesssim 4$

\section{ACKNOWLEDGMENTS}

We gratefully acknowledge the support and hospitality of the International Centre for Theoretical Physics (ICTP) where this work was done when the authors were visiting the center (B. T. under the Associate Scheme). We thank Dr. S. Moroni for discussions and for providing us with the Monte Carlo results. B. T. was partially supported by the Scientific and Technical Research Council of Turkey (TUBITAK) under Grant No. TBAG-AY/123.

\section{APPENDIX A: QUADRATURE FORMULA FOR $G(q, i \omega)$}

In this appendix we provide some details for obtaining a one-dimensional quadrature expression for $G(q, i \omega)$. The local-field factor is evaluated for frequencies on the imaginary axis, which greatly simplifies the self-consistent calculations.
The response function for the noninteracting inhomogeneous system is defined by

$$
\chi_{0}\left(\mathbf{q}, \mathbf{q}^{\prime} ; \omega\right)=\int d^{3} \mathbf{p} \frac{f\left(\mathbf{p}+\mathbf{q}^{\prime} / 2\right)-f\left(\mathbf{p}-\mathbf{q}^{\prime} / 2\right)}{\omega-\mathbf{p} \cdot \mathbf{q} / m+i \eta},
$$

which reduces to that of the homogeneous system, viz. $\chi_{0}(q, \omega)$, for $\mathbf{q}=\mathbf{q}^{\prime}$. Taking the distribution function as $f(\mathbf{p})=n \delta(\mathbf{p})$ for bosons at $T=0$, we obtain

$$
\chi_{0}\left(\mathbf{q}, \mathbf{q}^{\prime} ; \omega\right)=\frac{2 n \epsilon_{q q^{\prime}}}{(\omega+i \eta)^{2}-\epsilon_{q q^{\prime}}^{2}},
$$

where $\epsilon_{q q^{\prime}}=\mathbf{q} \cdot \mathbf{q}^{\prime} / 2 m$. Starting from Eq. (3) and using the above expression for $\chi_{0}$, the angular integration for $G(q, i \omega)$ is evaluated as

$$
\begin{array}{rl}
\int_{-1}^{1} & d \mu \frac{q^{2}-q k \mu}{\omega^{2}+\left(q^{2}-q k \mu\right)^{2}} \frac{1}{q^{2}+k^{2}-2 k q \mu} \\
= & -\frac{1}{2 q k\left(4 \omega^{2}+q^{4}-2 q^{2} k^{2}+k^{4}\right)}\left\{4 \omega \left[\tan ^{-1}\left(\frac{q(q-k)}{\omega}\right)\right.\right. \\
& \left.-\tan ^{-1}\left(\frac{q(q+k)}{\omega}\right)\right] \\
& +\left(k^{2}-q^{2}\right) \ln \left|\frac{\omega^{2}+q^{4}-2 q^{3} k+q^{2} k^{2}}{\omega^{2}+q^{4}+2 q^{3} k+q^{2} k^{2}}\right| \\
& \left.+2\left(q^{2}-k^{2}\right) \ln \left(\frac{q-k}{q+k}\right)^{2}\right\},
\end{array}
$$

in which the momentum variables $q$ and $k$ are scaled with $r_{0}$ and the energy variable $\omega$ is scaled with $1 / 2 m r_{0}^{2}$. Finally, the one-dimensional quadrature expression for $G(q, i \omega)$ takes the form

$$
G(q, i \omega)=-\frac{1}{3 \pi}\left(\omega^{2}+q^{4}\right) \int_{0}^{\infty} d k k^{2} P(k, q ; \omega)[S(k)-1],
$$

where $P(k, q ; \omega)$ is the result of the angular integral given by the right-hand side of Eq. (A3).
* On leave from Scuola Normale Superiore, I-56126 Pisa, Italy.

${ }^{1}$ D. Pines and P. Nozières, The Theory of Quantum Liquids (Benjamin, New York, 1966).

${ }^{2}$ R. Mincas, J. Ranninger, and S. Robaszkiewicz, Rev. Mod. Phys. 62, 113 (1990); A. S. Alexandrov and J. Ranninger, Solid State Commun. 81, 403 (1993); A. S. Alexandrov and N. F. Mott, Supercond. Sci. Technol. 6, 215 (1993).

${ }^{3}$ J.-P. Hansen, B. Jancovici, and D. Schiff, Phys. Rev. Lett. 29, 991 (1972); S. Schramm, K. Langange, and S. E. Koonin, Astrophys. J. 397, 579 (1992).

${ }^{4}$ K. S. Singwi, M. P. Tosi, R. H. Land, and A. Sjölander, Phys. Rev. 179, 589 (1968); K. S. Singwi and M. P. Tosi, Solid State Phys. 36, 177 (1981).
${ }^{5}$ Correlations in Electronic and Atomic Fluids, edited by P. Jena, R. Kalia, P. Vashishta, and M. P. Tosi (World Scientific, Singapore, 1990).

${ }^{6}$ A. A. Caparica and O. Hipólito, Phys. Rev. A 26, 2832 (1982); C. I. Um, W. H. Kahng, E. S. Yim, and T. F. George, Phys. Rev. B 41, 259 (1990); A. Gold, Z. Phys. B 89, 1 (1992); R. K. Moudgil, P. K. Ahluwalia, K. Tankeshwar, and K. N. Pathak, Phys. Rev. B 55, 544 (1997).

${ }^{7}$ S. Conti, M. L. Chiofalo, and M. P. Tosi, J. Phys.: Condens. Matter 6, 8795 (1994); M. L. Chiofalo, S. Conti, and M. P. Tosi, Mod. Phys. Lett. B 8, 1207 (1994).

${ }^{8}$ T. Hasegawa and M. Shimizu, J. Phys. Soc. Jpn. 38, 965 (1975); 39, 569 (1975). 
${ }^{9}$ G. Niklasson, in Electron Correlations in Solids, Molecules, and Atoms, edited by J. T. Devreese and F. Brosens (Plenum, New York, 1983), p. 99.

${ }^{10}$ A. Holas and S. Rahman, Phys. Rev. B 35, 2720 (1987); see also, K. S. Singwi, in Recent Progress in Many-Body Theories, edited by H. Kümmel and M. L. Ristig (Springer-Verlag, Berlin, 1984), p. 219.

${ }^{11}$ R. K. Moudgil, P. K. Ahluwalia, and K. N. Pathak, Phys. Rev. B 52, 11945 (1995).

${ }^{12}$ H. K. Schweng, H. M. Böhm, A. Schinner, and W. Macke, Phys. Rev. B 44, 13291 (1991).

${ }^{13}$ V. Apaja, J. Halinen, V. Halonen, E. Krotscheck, and M. Saarela,
Phys. Rev. B 55, 12925 (1997).

${ }^{14}$ G. Sugiyama, C. Bowen, and B. Alder, Phys. Rev. B 46, 13042 (1992).

${ }^{15}$ S. Moroni, S. Conti, and M. P. Tosi, Phys. Rev. B 53, 9688 (1996).

${ }^{16}$ C. F. Richardson and N. W. Ashcroft, Phys. Rev. B 50, 8170 (1994); Y. M. Malozovsky and J. D. Fan, Phys. Status Solidi B 201, 167 (1997).

${ }^{17}$ P. Vashishta and K. S. Singwi, Phys. Rev. B 6, 875 (1972).

${ }^{18}$ A. L. Fetter and J. D. Walecka, Quantum Theory of ManyParticle Systems (McGraw-Hill, New York, 1971).

${ }^{19}$ S. Ichimaru, Rev. Mod. Phys. 54, 1017 (1982). 